\title{
Cat Owners: How they Keep and Care for Their Own Cats and Their Attitudes to Stray and Feral Cats in Germany
}

\author{
Franziska Kuhne $^{1, ~ *}$, Kerstin Hoock ${ }^{1}$, Martin Kramer ${ }^{2}$, Hansjoachim Hackbarth ${ }^{3}$ \\ ${ }^{1}$ Working Group for Applied Ethology and Animal Behaviour Therapy, Department of Veterinary Clinical Sciences, Justus-Liebig-University \\ Giessen, Giessen, Germany \\ ${ }^{2}$ Small Animal Clinic - Surgery, Department of Veterinary Clinical Sciences, Justus-Liebig-University Giessen, Giessen, Germany \\ ${ }^{3}$ Foundation Institute for Animal Welfare and Farm Animal Behaviour, University of Veterinary Medicine Hannover, Hannover, Germany
}

\section{Email address:}

franziska.kuhne@vetmed.uni-giessen.de (F. Kuhne), Kerstin.Hoock@gmx.de (K. Hoock),

Martin.Kramer@vetmed.uni-giessen.de (M. Kramer), hackbarth@tierschutzzentrum.de (H. Hackbarth)

${ }^{*}$ Corresponding author

\section{To cite this article:}

Franziska Kuhne, Kerstin Hoock, Martin Kramer, Hansjoachim Hackbarth. Cat Owners: How they Keep and Care for Their Own Cats and Their Attitudes to Stray and Feral Cats in Germany. Animal and Veterinary Sciences. Vol. 7, No. 1, 2019, pp. 24-28.

doi: 10.11648/j.avs.20190701.14

Received: February 21, 2019; Accepted: March 3, 2019; Published: April 18, 2019

\begin{abstract}
Cats are among the most popular pets. A cat can be a feral cat, a stray cat, or a pet cat with variable degrees of free-roaming access to the outdoors. Free-roaming pet cats are the most significant source of cat overpopulation. Furthermore, if free-roaming cats do not wear identification, ownership is difficult to identify. Therefore, the aim of this study was to investigate 1) how pet cats are kept and cared for in Germany and 2) cat owners' attitudes to stray and feral cats and compliance with fertility control. The survey was designed as a multiple-choice questionnaire covering information on cat ownership and cat owners' attitudes to stray and feral cats and cat population management tools. Regression analyses were applied to determine whether the attitudes to stray and feral cats could be predicted from demographic parameters or one's own cat keeping. Many cat owners know stray and feral cats in their vicinity, but this knowledge has no influence on the spaying and neutering, identification, and registration status of their own cats and on their own cat keeping, e.g., with or without free access to the outdoors. Cat owners are afraid that stray and feral cats may transmit diseases to pet cats. The demographic parameters of the cat owners, e.g., their gender, age, and education level, significantly influenced their attitudes to keeping and caring for their own cat and controlling the cat population. Cat owners would accept the costs of legal requirements to spay or to neuter and to identify their own cat, and the majority would not relinquish their own cat to save the costs. To address the responsibility of cat owners is of utmost importance. It is necessary to spay or neuter and to identify free-roaming pet cats to prevent them from getting lost or pregnant. Each cat population management program's success depends on cat owners being involved and convinced of its necessity.
\end{abstract}

Keywords: Cat, Free-Roaming Cats, Fertility Control, Identification, Cat Overpopulation

\section{Introduction}

In modern society, cats are among the most popular pets. The Industrial Association of Pet Care Producers list the cat, with 13.4 million individuals, as the most popular pet in Germany [1]. Twenty-two percent of all households in Germany own at least one cat. The way of keeping catsalways or temporally indoors, with free or controlled access to the outdoors-determines the cat's physical and social environment. Local regulations, the safety of the urban area, the individual cat, and the experience of the cat owners influence the owners' decision on how to keep their cat. Cats need olfactory and chemical signals to evaluate their environment and use their own pheromones to mark familiar territory [2]. Keeping cats indoors might reveal behavior problems such as scratching, urine marking, and aggression in a multi-cat household. Cats kept outdoors are involved in territorial aggression or are attacked by animals, get lost, 
have unwanted litters, and get hit by cars.

Depending on the cat's level of socialization, ownership status, and lifestyle, a cat can be a pet cat with variable degrees of free-roaming access to the outdoors, a stray cat, or a feral cat [3-6]. A pet cat has an identifiable owner and home. These cats are kept indoors or have temporally or spatially free or controlled access to the outdoors. Freeroaming pet cats are a significant source of cat overpopulation if they are not spayed or neutered [3-4]. A stray cat is a cat who once lived in a home but has been lost or abandoned. Strays are usually tame and socialized with people but unclaimed [6]. On the other side, a feral cat is not socialized with people, lives as a single cat or in a colony, and avoids human contact. Feral cats are wild animals and are usually too fearful for handling or adoption by humans. Stray and feral cats mainly survive on their own but might be fed by caretakers [5-6].

For controlling or even reducing a cat population, the Trap-Neuter-Return (TNR) program has been launched in many communities [5, 7-9]. The goal of TNR is to trap all feral cats of a colony, to spay or neuter them, and to return them to their original territory. Allowing pet cats freeroaming access to the outdoors without non-surgical or surgical fertility control ignores cats' successful strategy of reproduction [10-15]. Cats reproduce quickly, but under normal circumstances, most offspring do not survive to adulthood. Cats with an owner or stray and feral cats being fed by caretakers survive and reproduce effectively. Therefore, fertility control is crucial to end cat overpopulation.

The implementation of programs to spay or neuter, to identify, and to register pet cats depends mainly on the compliance of pet cat owners. Therefore, the aim of this study was to investigate 1) how pet cats are kept and cared for in Germany and 2) cat owners' attitudes to stray and feral cats and compliance with fertility control of cats. Furthermore, we investigated whether there are an association between how pet cats are kept and cared for and cat owners' attitudes concerning TNR programs.

\section{Materials and Methods}

\subsection{Survey Design and Measures}

Cat owners were recruited to participate in this study on three ways: 1) an advertisement was placed in the magazine "Pfotenhieb" (addresses cat owners as a target group); 2) leaflets were laid out in 29 small animal clinics distributed all over Germany and located in rural areas and cities; and 3) members of two organizations (The German Farmers' Association and The German Association for agricultural Training) were informed via newsletters. The inclusion criterion for the study was actually being a cat owner.

The questionnaire covered information on the participants' cat ownership and their knowledge about the cat population, cat owners' familiarity with the cats' reproduction strategies, the possible influences of feral or stray cats on wildlife and human health, and participants' attitudes to programs to control the cat population. The survey was in German and designed as a multiple-choice questionnaire, though participants had the opportunity to add additional comments to some questions.

For a period of 12 months (between February 1, 2016, and January 31, 2017), the survey was posted on the Internet. Seven hundred and fifty-six participants submitted questionnaires. Only fully completed questionnaires were analyzed. Some questionnaires had to be excluded because respondents did not own a cat, resulting in a total sample size of 485 (64\% of 756$)$.

\subsection{Statistical Analysis}

Descriptive statistics were determined by use of SPSS $23^{\circledR}$ (SPSS Inc., Chicago, IL) to calculate summary statistics (means, percentages) for all binary variables. Logistic regression was generated to examine whether knowledge about stray and feral cats could be predicted from demographic parameters or one's own cat keeping. Data on participants' attitudes to stray and feral cats and how participants keep and care for their own cats, such as the number of cats, their fertility status, and possibility of access to the outdoors, were used as dependent variables. Demographic parameters, such as the gender and age of the participants, were treated as independent variables. One-way analysis of variance and univariate chi-squares were performed to ascertain if there were individual differences among the participants. The level of significance was set at $p<0.05$.

\section{Results}

The respondents were mainly women $(59.8 \%)$. A total of $32.8 \%$ of the respondents were aged between 41 and 50 years and $27.6 \%$ ranged from 51 to 60 years. The majority of respondents owned one cat $(36.6 \%)$ or two cats $(29.5 \%)$. Most pet cats were spayed or neutered (79.8\%), 46.5\% were identified, and $45.7 \%$ were registered. Women more often reported owning cats that are spayed or neutered $(\mathrm{F}=10.689$; $p<0.01)$, identified $(\mathrm{F}=45.884 ; p<0.01)$, and registered $(\mathrm{F}=58.516 ; p<0.01)$ than men. If their own cats were spayed or neutered, cat owners estimated the age of queens' sexual maturity more often at younger ages (mainly from four to eight month). Many pet cats were kept with free access to the outdoors (76.4\%). Furthermore, the more cats the respondents owned, the greater was the possibility for the cats to have access to the outdoors $(\mathrm{F}=5.174 ; p<0.01)$. Respondents who kept their cats with free access to the outdoors reported mainly that all of these cats were spayed or neutered $(78.9 \%)$. Nevertheless, $12.2 \%$ of these cats were fertile, $48.1 \%$ were not identified, and $48.6 \%$ were not registered. The gender of respondents best predicted their own cat care-giving relating to spaying and neutering, to identifying, and to registering in that mainly women kept their pet cats that way.

The respondents estimated the number of stray and feral 
cats in Germany mainly from 10,000 to $100,000(33.4 \%)$ and from 100,000 to 1 million $(31.5 \%)$. Two-thirds of the respondents $(69.9 \%)$ know stray or feral cats in their vicinity, and $20.9 \%$ of them feel disturbed from these cats. Many of the respondents think that stray and feral cats do not get sufficient medical care $(85.2 \%)$, and nearly half of them believe that stray and feral cats do not have enough to eat $(47.6 \%)$ or are doing badly $(46.4 \%)$. Women believe more than men do that stray and feral cats are doing badly $(\mathrm{F}=18.547 ; p<0.01)$, do not have enough shelter $(\mathrm{F}=16.958$; $p<0.01)$, do not get enough to eat $(\mathrm{F}=20.391 ; p<0.01)$, and do not get sufficient medical care $(\mathrm{F}=28.317 ; p<0.01)$.

One-third of the respondents think $(31.8 \%)$ that stray and feral cats have an influence on the environment, particularly through hunting birds and small mammals. Fewer of them $(19.4 \%)$ are afraid that stray and feral cats transmit diseases to humans, but many believe $(75.3 \%)$ that stray and feral cats might transmit diseases to pet cats. The effects of demographic parameters on the respondents' opinions on stray and feral cats and their impact on the environment are depicted in Table 1.

Table 1. Effects of demographic parameters on respondents'opinions on stray and feral cats and methods to reduce cat overpopulation (significant effects of logistic regression are presented).

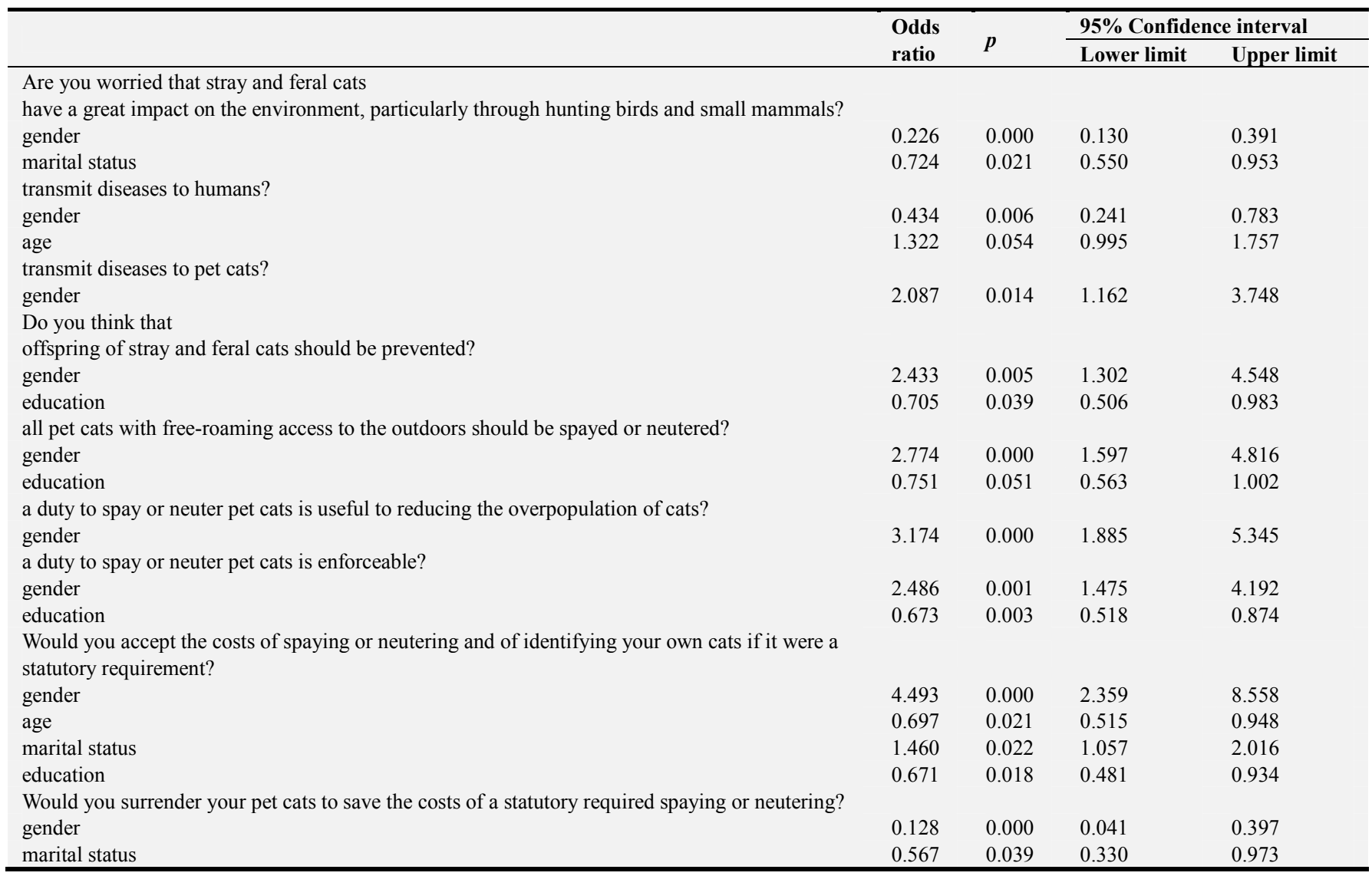

The respondents' estimation of the number of stray and feral cats was significantly associated with their knowledge of stray or feral cats in their vicinity $(\mathrm{F}=23.238 ; p<0.01)$, in that the more the people are personally concerned, the higher they estimated the number of stray and feral cats. If respondents are informed about the animal welfare concerns of stray or feral cats, they have significantly more often spayed or neutered $(\mathrm{F}=5.172 ; p<0.05)$ and identified $(\mathrm{F}=5.659 ; p<0.05)$ their own cats (but with no significant change in registration). The knowledge of stray or feral cats in the vicinity had no influence on the fertility, identification, and registration status of owned cats and on one's own cat keeping, e.g., with or without free access to the outdoors.

The demographic parameters of the respondents, e.g., their gender, age, marital status, and education level, significantly influenced their opinion on cat population management tools
(Table 1). Women believe more than men do that offspring of feral cats should be prevented and that all cats with freeroaming access to the outdoors should be spayed or neutered. Furthermore, there was a significant influence of the respondents' gender on their answer to the statements "a duty to spay or neuter pet cats is useful to reducinge the population of cats" and "a duty to spay or to neuter pet cats is enforceable" in that women agreed more often to these statements. Generally, $62.1 \%$ of the cat owners believe in the success of a duty to spay or to neuter, to identify, and to register pet cats. If it were a legal requirement, $76.2 \%$ of the cat owners would accept the costs for spaying or neutering and for identifying their own cat, but women would agree to the costs more often than men would (Table 1). A relinquishment of the owned cat to save the costs of a legally required spaying or neutering and identification was no 
option for $92.0 \%$ of the cat owners; however, men would more often relinquish their cats.

\section{Discussion}

The aim of this study was to investigate how people in Germany keep and care for their owned cats and, secondarily, whether there are factors which influence cat owners' attitudes to TNR programs. The demographic parameters of the cat owners, e.g., their gender, age, marital status, and education level, significantly influenced their attitudes to handling their own cat and controlling the cat population.

The data in this study are based on a self-selected survey. The cat owners chose to take part in the survey; thus, selfselection bias might arise. There are many possible reasons, e.g., motivation, socioeconomic status, or prior test-taking experience, why mainly female cat owners participated in the survey. The majority of cat owners kept one or two cats, which is slightly less than reported in other studies [16]. The own cats are more often spayed or neutered if the cat owners are familiar with the well-being concerns of stray and feral cats. The majority of the cat owners believe that stray and feral cats are doing badly, do not get sufficient medical care, and have insufficient food or shelter but have no serious impact on the environment through hunting birds and small mammals.

It is estimated that $70 \%$ of feline admissions to animal shelters are euthanized in the U.S. [17]. The euthanasia of healthy cats, equally of stray and feral cats, is forbidden in Germany ( $§ 1$ German Animal Welfare Act) [18]' but it is allowed by law that municipalities can establish population management programs. To reduce an overpopulation of cats, the trap-neuter-return (TNR) program has been successfully established in many countries [5, 17, 19-20]. Different methods of surgical or non-surgical contraception, health control, permanent identification, and return/release programs have been launched and analyzed [7, 14, 21-22], Kalz [23] found that the population density decreased if all females of a population were spayed and tomcats stayed uncastrated. The majority of the respondents to this study agreed that all pet cats with free-roaming access to the outdoors should be spayed or neutered. Three-fourths of the respondents would accept the costs of legal requirements to castrate and identify their own cat, and the majority (92.0\%) would not relinquish owned cats to save on these costs. Nevertheless, the respondents' views on population management tools depended on their gender, age, marital status, and education. These demographic parameters and the intrinsic value of cats must be considered if campaigns are launched to raise awareness of the health and welfare problems of stray and feral cats.

Many respondents underestimated the number of stray and feral cats in Germany. It has been estimated that there are nearly two million stray and feral cats in Germany [24], but the true number of these cats is unknown and difficult to estimate. The development of feline intake and euthanasia at shelters, the number of cats fed by local caretakers, the number of car accidents, and the population of wild-living small mammals and birds are commonly used to estimate the number of stray and feral cats $[3,9,14,17]$. The cat owners of this study are afraid that stray and feral cats transmit diseases to pet cats, but their impact on wild-living small mammals and birds is, in their opinion, negligible. Mainly women believe that stray and feral cats are doing badly, have not enough shelter, get not enough to eat, and do not get sufficient medical care. Nevertheless, stray and feral cats in the vicinity have no influence on their keeping and caring for their own cat e.g., its reproduction status and access to the outdoors. This discrepancy between one's own concerns for the well-being of the stray and feral cats themselves and one's own keeping of cats may be based on the difficulty of assessing the true impact of free-roaming pet cats on the environment and on stray and feral cats, which is often fueled by emotional arguments and without scientific data [9]. Only $38 \%$ of the cat owners in this study believe that a legal requirement would be enforceable, which means that to address the responsibility of cat owners by educational efforts, these opinions are of utmost importance [10]. To rely on the kind-heartedness of caretakers and people who provide food and shelter for stray and feral cats ignores the responsibility of each cat owner. Like for indoor pet cats, which are often spayed or neutered to prevent unwanted pregnancy and active fighting and to reduce indoor spraying and altercations over food or territory [25-27], it is necessary to spay or neuter and identify free-roaming pet cats to prevent them from getting lost or pregnant.

\section{Conclusion}

The major finding of this study is that cat owners know stray and feral cats in their vicinity but continue to allow their fertile cats free access to the outdoors. The majority of the cat owners surveyed would not relinquish their own cat to save the costs of a legal requirement to spay or to neuter and to identify their own cat. Further research is needed to understand the discrepancy between cat owners' attitudes to stray and feral cats and cat population management tools and their keeping and caring for their own cats.

\section{Acknowledgements}

The authors would like to express their gratitude to the cat owners for participating in this study. We also thank Julia Steinkamp, Kathrin Roiner, Katrin Steinert, Wiebke Wolkenhauer, and Kerstin Rolinger for their encouragement and insightful feedback.

\section{References}

[1] Industrial Association of Pet Care Producers' (The German Pet Market, https://www.ivh-online.de/en/theassociation/facts-figures.html (2019, accessed February 17, 2019). 
[2] Ellis, S. L. H., I. Rodan, H. C. Carney, S. Heath, I. Rochlitz and L. Shearburn (2013) AAFP and ISFM feline environmental needs guidelines. Journal of Feline Medicine and Surgery 15, 219-230.

[3] Scott, K. C., J. K. Levy JK and P. C. Crawford (2002) Characteristics of free-roaming cats evaluated in a trap-neuterreturn program. Journal of the American Veterinary Medical Association 221, 1136-1138.

[4] Centonze, L. A. and J. K. Levy (2002) Characteristics of freeroaming cats and their caretakers. Journal of the American Veterinary Medical Association 220, 1627-1633.

[5] Robertson, S. A. (2008) A review of feral cat control. Journal of Feline Medicine and Surgery 10, 366-375.

[6] Gosling, L., J. Stavisky and R. Dean (2013) What is a feral cat?: Variation in definitions may be associated with different management strategies. Journal of Feline Medicine and Surgery $15,759-764$.

[7] Benka, V. A. W. (2015) Ear tips to ear tags: Marking and identifying cats treated with non-surgical fertility control. Journal of Feline Medicine and Surgery, 17, 808-815.

[8] Wallace, J. L. and J. K. Levy (2006) Population characteristics of feral cats admitted to seven trap-neuter-return programs in the United States. Journal of Feline Medicine and Surgery 8, 279-284.

[9] Levy, J. K. and P. C. Crawford (2004) Humane strategies for controlling feral cat populations. Journal of the American Veterinary Medical Association 225, 1354-1360.

[10] Murray, J. K., J. R. Mosteller, J. M. Loberg, M. Andersson and V. A. W. Benka (2015) Methods of fertility control in cats: Owner, breeder and veterinarian behavior and attitudes. Journal of Feline Medicine and Surgery 17, 790-799.

[11] Briggs, J. (2015) Non-surgical fertility control: Current and future options for cat health and welfare. Journal of Feline Medicine and Surgery 17, 740-741.

[12] Rhodes, L. (2017) New approaches to non-surgical sterilization for dogs and cats: Opportunities and challenges. Reproduction in Domestic Animals 52 Suppl 2, 327-331.

[13] Johnston, S. and L. Rhodes (2015) No surgery required: The future of feline sterilization: An overview of the Michelson Prize \& Grants in Reproductive Biology. Journal of Feline Medicine and Surgery 17, 777-782.

[14] Boone, J. D. (2015) Better trap-neuter-return for free-roaming cats: Using models and monitoring to improve population management. Journal of Feline Medicine and Surgery 17, 800-807.
[15] Little, S. (2011) Feline reproduction: Problems and clinical challenges. Journal of Feline Medicine and Surgery 13, 508515 .

[16] Strickler, B. L. and E. A. Shull (2014) An owner survey of toys, activities, and behavior problems in indoor cats. Journal of Veterinary Behavior: Clinical Applications and Research 9, 207-214.

[17] Spehar, D. D. and P. J. Wolf (2018) The Impact of an Integrated Program of Return-to-Field and Targeted TrapNeuter-Return on Feline Intake and Euthanasia at a Municipal Animal Shelter. Animals 8, 55.

[18] BMELF. German Animal Welfare Act: TierSchG (Tierschutzgesetz), 2017.

[19] Levy, J. K., D. W. Gale and L. A. Gale (2003) Evaluation of the effect of a long-term trap-neuter-return and adoption program on a free-roaming cat population. Journal of the American Veterinary Medical Association 222, 42-46.

[20] Neville, P. F. and J. Remfry (1984) Effect of neutering on two groups of feral cats. Veterinary Record 114, 447-450.

[21] Kutzler, M. and A. Wood (2006) Non-surgical methods of contraception and sterilization. Theriogenology 66, 514-525.

[22] Foley, P., J. E. Folet, J. K. Levy and T. Paik (2005) Analysis of the impact of trap-neuter-return programs on populations of feral cats. Journal of the American Veterinary Medical Association 227, 1775-1781.

[23] Kalz, B (2001) Populationsbiologie, Raumnutzung und Verhalten verwildeter Hauskatzen und der Effekt von Maßnahmen zur Reproduktionskontrolle Doctoral Thesis, Humboldt-Universität zu Berlin, doi: 10.18452/14587.

[24] Deutscher Tierschutzbund. Katzenschutz-Kampagne: Die Straße ist grausam. Kastration harmlos., https://www.tierschutzbund.de/aktion/kampagnen/heimtiere/k atzenschutz-kampagne/ (2016, accessed September 3, 2018).

[25] Hart, B. L. (1991) Effects of neutering and spaying on the behaviour of dogs and cats: Questions and answers about practical concerns. Journal of the American Veterinary Medical Association 198, 1204-1205.

[26] Spain, C. V., J. M. Scarlett and S. M. Cully (2002) When to neuter dogs and cats: a survey of New York state veterinarians' practices and beliefs. Journal of the American Animal Hospital Association 38, 482-488.

[27] Barry, K. J. and S. L. Crowell-Davis (1999) Gender differences in the social behavior of the neutered indoor-only domestic cat. Applied animal behaviour science 64, 193-211. 